\title{
Quercetin improves lipid metabolism via SCAP-SREBP2-LDLr signaling pathway in early stage diabetic nephropathy
}

This article was published in the following Dove Press journal: Diabetes, Metabolic Syndrome and Obesity: Targets and Therapy

\author{
Xiyuan Jiang ${ }^{1,2}$ \\ Jiangyi $\mathrm{Yu}^{3}$ \\ Xin Wang ${ }^{3}$ \\ Jing $\mathrm{Ge}^{3}$ \\ $\mathrm{Nan} \mathrm{Li}^{1}$
}

\begin{abstract}
'The First Clinical Medical School, Nanjing University of Chinese Medicine, Jiangsu 210029, People's Republic of China; ${ }^{2}$ Eodocrinology Department, KunShan Affiliated Hospital of Nanjing University of Chinese Medicine, Jiangsu, 215300, People's Republic of China; ${ }^{3}$ Eodocrinology Department, Affiliated Hospital of Nanjing University of Chinese Medicine, Jiangsu 213003, People's Republic of China
\end{abstract}

Purpose: Quercetin, the most widely distributed flavonoid, has been shown to have multiple properties and beneficial effects on various metabolic diseases. Thus, our aim was to investigate the underlying mechanism whereby quercetin regulates renal lipid accumulation and ameliorates early diabetic renal injuries in $\operatorname{Lepr}^{\mathrm{db}} / \operatorname{Lepr}^{\mathrm{db}}(\mathrm{db} / \mathrm{db})$ mice, a model of type 2 diabetes.

Methods: $\mathrm{db} / \mathrm{db}$ mice were administered either $50 \mathrm{mg} / \mathrm{kg}$ or $100 \mathrm{mg} / \mathrm{kg}$ quercetin by oral gavage once a day to evaluate its effects on early stage diabetic nephropathy; mice were sacrificed at the end of the 10th week after intervention; a similar number of $\mathrm{db} / \mathrm{db}$ and $\mathrm{db} / \mathrm{m}$ mice were used as controls. During the experimental study, the general status of the animals was observed daily; body weight and blood glucose concentrations were measured at bi-weekly intervals. Biochemical parameters of lipid metabolism were measured by automatic biochemical analyzer. Renal function parameters were performed using commercial kits. Early renal histological changes and lipid accumulation were demonstrated by H\&E staining and Oil-Red-O staining, respectively. Moreover, the expression of key proteins in the low-density lipoprotein receptors (LDLr)-SREBP-2-SREBP cSCAP signaling pathway in the kidneys of diabetic mice was detected by Western blot assay.

Results: Compared with diabetic controls, quercetin not only ameliorated albuminuria and urinary albumin-to-creatinine ratio, but also decreased blood urea nitrogen and glucose, serum cholesterol, triglycerides, and low-density lipoprotein cholesterol, whereas it had no remarkable effect on the high-density lipoprotein cholesterol in diabetic $\mathrm{db} / \mathrm{db}$ mice. Additionally, the evidently down regulated expression of LDLr, HMGCR, SREBP-2, and SCAP subsequently attenuated the renal lipid profile change and lipid droplet accumulation, resulting in the alleviation of renal injury of $d b / d b$ mice.

Conclusion: Quercetin safely and efficiently alleviates early diabetic renal injuries, possibly through improving the lipid metabolism via SCAP-SREBP2-LDLr signaling pathway.

Keywords: quercetin, diabetic nephropathy, lipid metabolism, renal function, low density lipoprotein receptor

\section{Introduction}

Prevalence of diabetes mellitus (DM) is growing world-wide and it is expected to affect almost 600 million people by $2035 .{ }^{1} \mathrm{DM}$ and its complications are a leading cause of incapacity and death world-wide. Diabetic nephropathy (DN), one of the most serious microvascular complications of DM, develops in more than $40 \%$ of type 1 and 2 diabetic patients, and it has become a mounting global menace. ${ }^{2}$ Previous studies have shown that genetic susceptibility is an important factor in $\mathrm{DN}$, and exposure of renal tissues to chronic hyperglycemia seems to be the
Correspondence: Jiangyi Yu

Eodocrinology Department, Affiliated Hospital of Nanjing University of Chinese Medicine, Jiangsu 213003, People's Republic of China

Email yujiangyi2007@I63.com 
initiating factor, ${ }^{3}$ acting through multiple pathogenic pathways including the formation of advanced glycation end products, ${ }^{4}$ hemodynamic and metabolic alterations, oxidative stress, and activation of the renin-angiotensin system. ${ }^{5,6}$ More recently, there has been growing evidence that abnormal lipid metabolism and renal lipid accumulation play a role in the pathogenesis of DN. ${ }^{7}$ Additionally, numerous studies on diabetic animal models and patients have found that ectopic lipid accumulation in the fatty kidney is associated with obesity-related DN. ${ }^{8,9}$ Clearly, there is a need for more studies in mice and other animal models with relevance for diabetic kidney changes in patients with $\mathrm{DM}$. The $\mathrm{db} / \mathrm{db}$ mouse is a rodent model with genetic DM that may be used to study diabetic dyslipidemia and kidney changes in DM, it is characterized by being overweight, having dyslipidemia and the risk to develop diabetic complications. ${ }^{10}$ However, so far, relatively little progress has been made in the treatment of early stage DN. ${ }^{11}$ Therefore, it is necessary to understand the mechanism of pathophysiology of DN, so that we can develop new therapies to help patients with DM. ${ }^{12}$

Cholesterol biosynthesis and uptake are important pathways that are required for cell maintenance, because cholesterol is an essential lipid that is important for maintaining membrane fluidity and raft formation, as well as steroid and bile acid syntheses. ${ }^{13}$ It is well-known that HMGCR is the key enzyme for cholesterol synthesis ${ }^{14}$ and low density lipoprotein receptor (LDLr) is the mediator of cholesterol uptake, ${ }^{15}$ the two key proteins which are predominantly regulated by SREBP-2. When cells lack or contain excess cholesterol relative to that required for their physiological needs, the up- or down-regulation of HMGCR and LDLr is mediated via a negative feedback mechanism that is tightly controlled by the other two proteins, SREBP-2 and SREBP SCAP, ${ }^{6}$ which are important for keeping a balance of cholesterol at cell and systemic level. Notably, once the tight SCAP-SREBP2-LDLr negative feedback system is disrupted by damage factors, the increasing HMGCR-mediated cholesterol synthesis and LDLr-mediated cholesterol uptake may exacerbate cholesterol accumulation in kidneys, ${ }^{16}$ subsequently causing renal injuries mediated by lipids. Accordingly, targeting the activation of SCAP-SREBP2-LDLr signaling pathway and the expression of HMGCR in the kidneys, could be therapeutic mechanisms for ameliorating early stage DN. In China, traditional Chinese medicine (TCM) has been widely used in the treatment of DM and its complications for a long time, it possesses a lot of advantages compared to conventional medical approaches in the prevention of diabetic complications due to less toxicity and/or side effects. ${ }^{17,18}$ As a well-known TCM, quercetin $\left(3,3^{\prime}, 4^{\prime}, 5,7\right.$-pentahydroxyflavone), the most widely distributed flavonoid, is ubiquitously present in regular diets and exhibits a range of biological properties and health-promoting effects, ${ }^{19-22}$ which are closely associated with its beneficial actions against various metabolic diseases including glucose and lipid metabolism disorder and insulin resistance. ${ }^{23}$ Jeong et al reported that quercetin could be effective in improving hyperglycemia, dyslipidemia, and antioxidant status in type $2 \mathrm{DM}^{24}$ Despite pharmacological interest in quercetin and its possible nutritional benefits, up to present, the molecular mechanisms underlying its chemopreventive activity remain poorly understood and the precise effect of quercetin on diabetic renal lipid accumulation has not been previously reported. Considering that lipid metabolism disorder is one of the leading causes of DM-related complications, we aimed to explore the protective effect of quercetin on early stage DN using a type 2 diabetic mouse model and to further clarify the underlying molecular mechanisms, especially those associated with SCAP-SREBP2-LDLr signaling pathway.

\section{Methods}

\section{Chemicals and reagents}

Quercetin (purity $\geq 95 \%$, high performance liquid chromatography) purchased from Sigma-Aldrich Co. (St Louis, MO, USA) was dissolved in DMSO and the dosages of quercetin chosen were based on previous reports. ${ }^{23,25,26}$ Commercial kits for urine albumin, urine creatinine, blood urea nitrogen, and serum creatinine were purchased from Jiancheng Biotechnology Company (Nanjing, China). Total cholesterol, triglycerides, low-density lipoprotein cholesterol (LDL-C), and high-density lipoprotein cholesterol (HDL-C) kits were purchased from Shanghai Roche (Shanghai, China). Total protein extraction kit and bicinchoninic acid protein assay kit were provided by Jiangsu KeyGEN BioTECH Corp., Ltd. (Nanjing, China). Antibodies including HMGCR, LDLr, SREBP-2, SCAP, and $\beta$-actin were purchased from Santa Cruz Biotechnology, Inc. (Dallas, TX, USA). Other chemicals were of analytical grade and supplied by local reagent retailer.

\section{Animals and experimental design}

Six-week-old Lepr ${ }^{\mathrm{db}} /$ Lepr $^{\mathrm{db}}$ (db/db) male mice (C57BL/KsJ background) and their age-matched non-diabetic $\mathrm{db} / \mathrm{m}$ mice 
were obtained from Nanjing Biomedical Research Institute of Nanjing University (Nanjing, China). Animals were housed in polypropylene cages and permitted access to food and water ad libitum at a temperature range of $20^{\circ} \mathrm{C}-23^{\circ} \mathrm{C}$ on a $12 / 12$-hour light-dark cycle. All protocols carried out were approved by the Animal Research Ethics Committee of Nanjing University of Chinese Medicine, Jiangsu, China (ethics no: ACU170404). The study was conducted according to the regulations for animal experimentation issued by Animal Research Ethics Committee of Nanjing University of Chinese Medicine.

After 1 week of adaptation, the $\mathrm{db} / \mathrm{db}$ mice were divided into three groups ( $\mathrm{n}=8$ for each group): diabetic control: mice were administered only placebo (saline) (50 mg/kg/day); low dose quercetin: mice were administered quercetin at $50 \mathrm{mg} / \mathrm{kg} /$ day; and high dose quercetin: mice were administered quercetin at $100 \mathrm{mg} / \mathrm{kg} / \mathrm{day}$. The mice were treated with quercetin (approximately $0.2 \mathrm{~mL}$ in volume) by oral gavage once a day at 9:00-10:00 am. Meanwhile, the $\mathrm{db} / \mathrm{m}$ mice were used as non-diabetic controls. After administration for 10 weeks, the mice were housed in metabolic cages (Fengshi Inc., Suzhou, JS, China) for urine and blood collection. Thereafter, the mice were sacrificed and kidneys were immediately excised and weighed. Part of the renal tissue was fixed in 4\% paraformaldehyde and the remaining tissues were stored at $-80^{\circ} \mathrm{C}$ for later analysis.

\section{General status and biochemical parameters}

General status including the spirit, eating, drinking, fur colour, and activities of mice were observed daily, and body weight and blood glucose concentrations were measured at bi-weekly intervals for all animals.

Renal function parameters including urine albumin and creatinine in 24-hour urine samples, as well as blood urea nitrogen and serum creatinine were measured using commercial kits (Jiancheng Biotechnology Company, Nanjing, China) according to the manufacturer's instructions. The 24-hour urine total protein (UTP) was calculated as urine protein concentration $(\mu \mathrm{g} / \mathrm{mL})$ multiplied by 24 -hour urine volume $(\mathrm{mL})$, and urinary albumin-to-creatinine ratio (UACR) $(\mu \mathrm{g} / \mathrm{mg})$ was calculated as urine albumin concentration divided by the corresponding spot urine creatinine concentration, as per our previous study. ${ }^{27}$ The kidney index $(\mathrm{mg} / \mathrm{g})$ was calculated as the ratio of two kidneys' weight and the body weight of each mouse.

Biochemical parameters of lipid metabolism including serum cholesterol, triglycerides, LDL-C, and HDL-C concentration were measured by automatic biochemical analyzer in the Department of Laboratory Medicine, Affiliated Hospital of Nanjing University of Traditional Chinese Medicine. $^{28}$

\section{Renal histopathological analysis}

Renal tissues from mice fixed in 4\% paraformaldehyde were dehydrated with a graded series of alcohol and then embedded in paraffin. Sections of $5 \mu \mathrm{m}$ were deparaffinized, rehydrated, and stained with H\&E solution.

To directly observe the renal lipid accumulation, OilRed-O staining was performed according to our previously study. ${ }^{16}$ Briefly, frozen sections were cut at $10 \mu \mathrm{m}$ thick, air dried for 1 hour, and fixed in 4\% paraformaldehyde for 1 minute. The sections were rinsed with distilled water and stained in the Oil-Red-O working solution (Sigma-Aldrich Co.) for 30 minutes, then rinsed again for 1 minute in $4 \%$ paraformaldehyde and returned to distilled water. Thereafter, samples were counter-stained with hematoxylin, and lipid droplets in cells and tissues appeared as red spots. One hundred photographs of non-overlapping sections were randomly selected and all these data were calculated with Image-Pro Plus 6.0 software (Media Cybernetic).

\section{Western blot assay}

Frozen half kidneys were homogenized and sonicated in lysis buffer as previously described. ${ }^{29}$ After separation, lysate proteins were separated on 7.5\% (wt/vol) SDS-PAGE gel and subsequently electroblotted to PVDF membrane. After blocking of non-specific binding sites with 5\% non-fat milk, the blotted membranes were washed and then incubated separately with primary rabbit polyclonal antibodies against HMGCR (1:2,000), LDLr (1:1,000), SREBP-2 (1:1,000), $\operatorname{SCAP}(1: 1,000)$, and $\beta$-actin $(1: 2,000)$ overnight at $4^{\circ} \mathrm{C}$. After washing, the membranes were incubated at room temperature for 30 minutes with appropriate secondary antibodies. Signal was quantified by chemiluminescence detection using the LabWorks software (UVP Laboratory Products, Upland, CA, USA), and normalized to internal loading control ( $\beta$-actin) density.

\section{Statistical analysis}

Data were presented as means \pm SEM. Statistical differences between groups were detected using Student's $t$-test or ANOVA followed by Tukey's test with Statistical Package for Social Science (version 18.0; SPSS, Inc., Chicago, IL, USA). $P$-value less than 0.05 was considered statistically significant. 


\section{Results}

\section{Effect of quercetin on general status}

During the whole experiment period, administration of either $50 \mathrm{mg} / \mathrm{kg}$ or $100 \mathrm{mg} / \mathrm{kg}$ quercetin daily to mice did not cause any clinical symptoms of toxicity. Food intake and liquid consumption of $d b / d b$ mice were significantly increased as compared with $\mathrm{db} / \mathrm{m}$ mice $(P<0.01$, Figure $1 \mathrm{~A}$ and $\mathrm{B})$, but there was no significant difference in $\mathrm{db} / \mathrm{db}$ mice treated with or without quercetin (Figure 1A and B), indicating that quercetin did not exert a marked effect on food and liquid consumption of $\mathrm{db} / \mathrm{db}$ mice. Additionally, all mice displayed a sustained increase in body weight during the course of the study, the body weight of $\mathrm{db} / \mathrm{db}$ mice was obviously higher compared with $\mathrm{db} / \mathrm{m}$ mice $(P<0.01$, Figure $1 \mathrm{C})$, and the body weight of $\mathrm{db} / \mathrm{db}$ mice was slightly lower upon quercetin supplementation compared with that of $\mathrm{db} / \mathrm{db}$ controls, but not to a significant extent (Figure 1C). Whereas the body weight gains of $\mathrm{db} / \mathrm{db}$ mice were obviously higher than those of $\mathrm{db} /$ $\mathrm{m}$ mice at the end of study $(P<0.05$ or $P<0.01$, Figure 1D). More importantly, the body weight gains of $\mathrm{db} / \mathrm{db}$ mice were lower in quercetin-treated group than those of $\mathrm{db} / \mathrm{db}$ controls $(P<0.05$, Figure 1D), but there was no significant difference between low and high doses of quercetin-treated groups (Figure 1D), suggesting the lowering effect of quercetin on body weight gains in diabetic mice.

\section{Effect of quercetin on blood glucose concentration}

Blood glucose concentration of non-diabetic control mice remained fairly constant during the 10-week period (Figure 2). The level of blood glucose increased in an agedependent manner in $\mathrm{db} / \mathrm{db}$ control mice and the
A

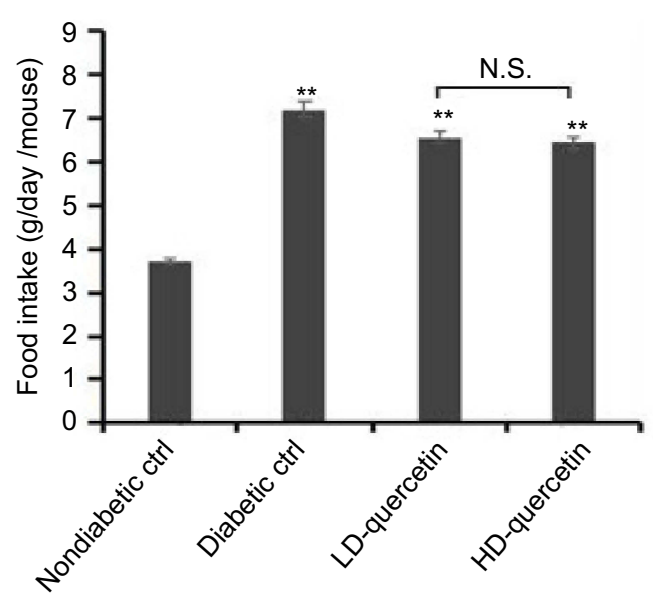

C

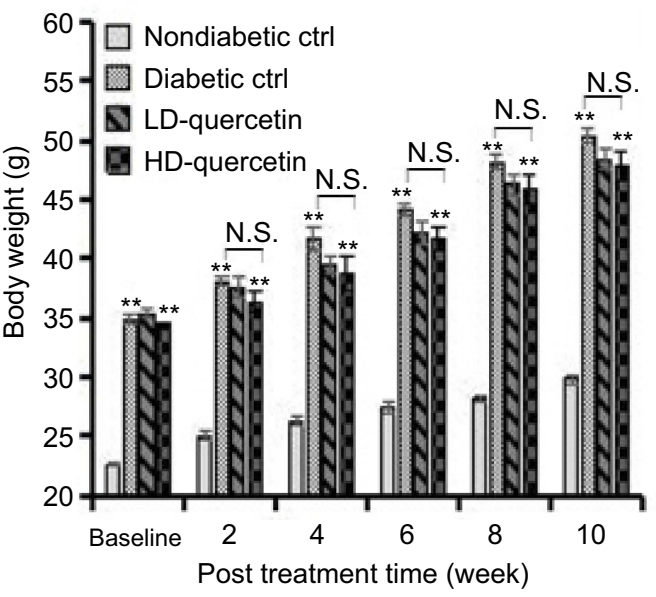

B

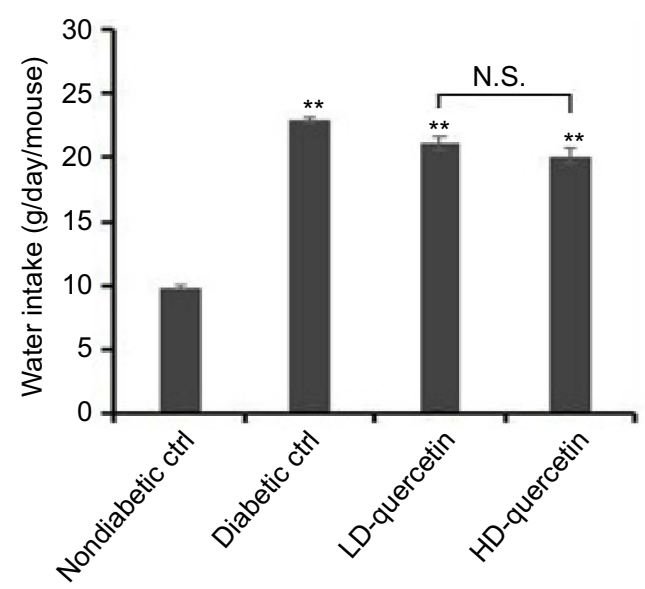

D

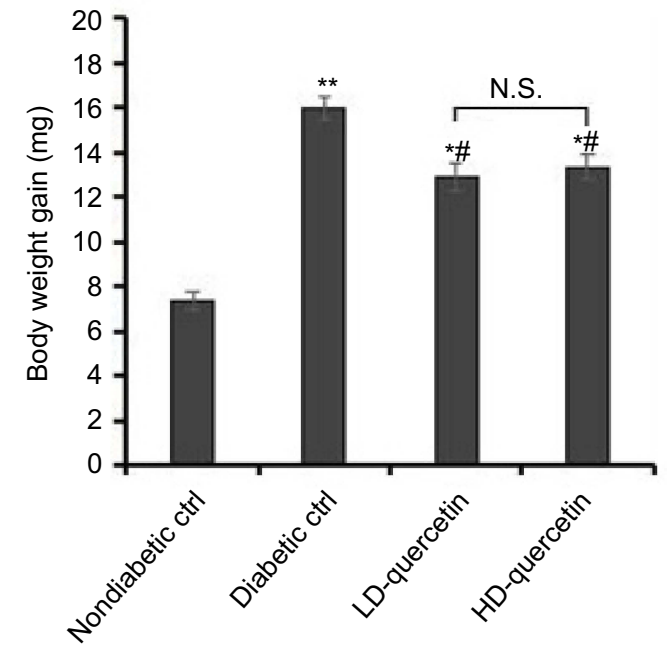

Figure I Effect of quercetin on food and water consumption and body weight of mice after administration for 10 weeks. (A) Daily food intake of mice; (B) daily water intake of mice; (C) body weight of mice measured at bi-weekly intervals; (D) body weight gains of mice. $* P<0.05$ or $* * P<0.0$, compared with non-diabetic control group; ${ }^{\#} P<0.05$, compared with diabetic control groups.

Abbreviations: LD-quercetin, low dose quercetin; HD-quercetin, high dose quercetin; NS, no significant comparison; ctrl, control. 


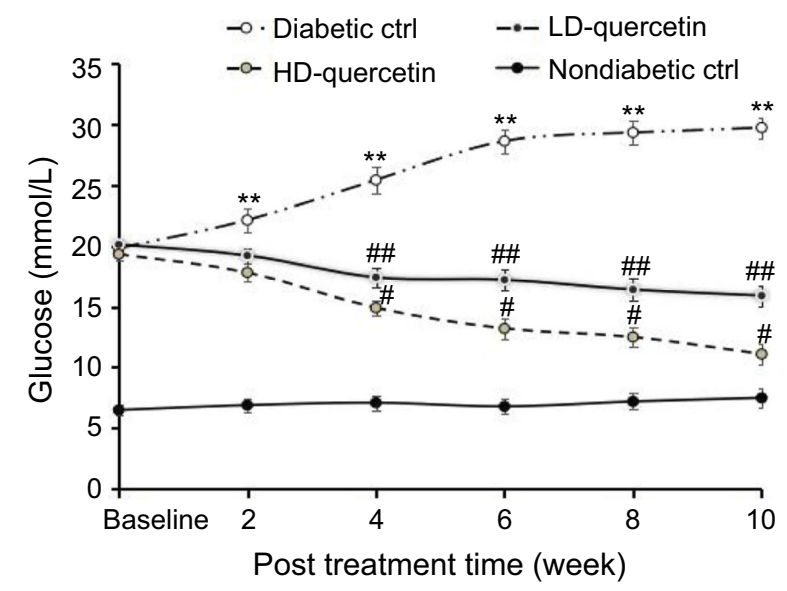

Figure 2 Effect of quercetin on the blood glucose concentration of mice after administration for 10 weeks. ${ }^{*} * \# P<0.01$ or ${ }^{\#} P<0.05$, compared with non-diabetic control group; ${ }^{\#} P<0.05$ or ${ }^{\#} P<0.01$, compared with diabetic control groups; ${ }^{\#} P<0.05$, compared with LD-quercetin group.

Abbreviations: LD-quercetin, low dose quercetin; HD-quercetin, high dose quercetin; ctrl, control.

hyperglycemia was maintained throughout the 10-week period. The increase of blood glucose was significantly attenuated in $\mathrm{db} / \mathrm{db}$ mice treated with either low or high doses of quercetin compared with $\mathrm{db} / \mathrm{db}$ control mice, but the glucose levels were still significantly higher than those observed in non-diabetic controls $(P<0.05$, Figure 2$)$, confirming that quercetin shows a very significant effect on lowering blood glucose level.

\section{Effect of quercetin on the serum creatinine and blood urea nitrogen}

The levels of serum creatinine and blood urea nitrogen in diabetic mice were obviously higher than those in nondiabetic controls $(P<0.01$, Figure 3$)$, and the higher levels of serum creatinine and blood urea nitrogen decreased dramatically in diabetic mice treated with high dose quercetin as compared with either low dose quercetin or diabetic control groups $(P<0.05$, Figure 3$)$, indicating that quercetin shows a remarkable amelioration of renal dysfunction in diabetic mice.

\section{Effect of quercetin on urinary albumin excretion}

Early stage DN is characterized by hyperfiltration and microalbuminuria. Thus, the effect of quercetin on the UTP and UACR were evaluated in DN mice. It was indicated that the DM-related increases of UTP and UACR were attenuated in diabetic mice with an increasing dose of quercetin, as compared with diabetic controls $(P<0.01$, Figure $4 \mathrm{~A}$ and $\mathrm{B})$, but the

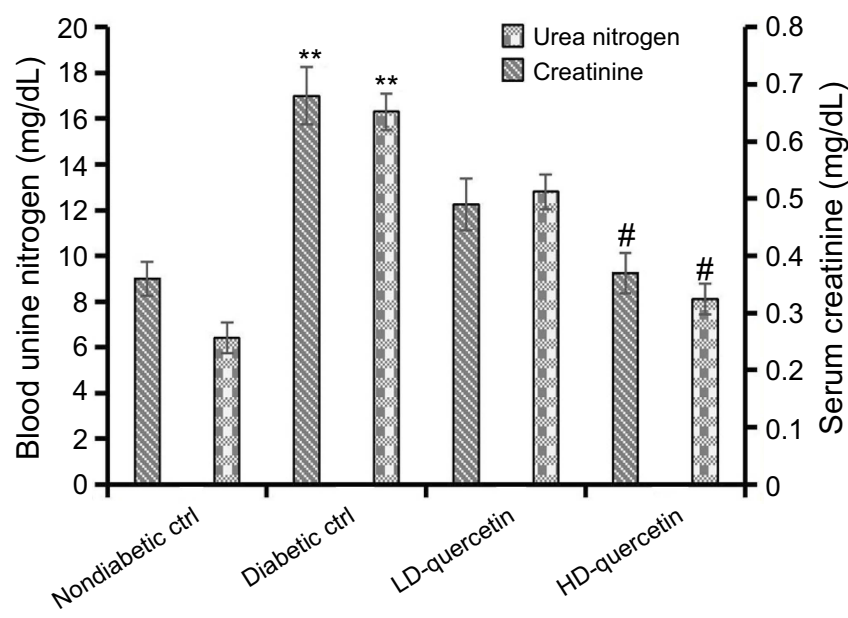

Figure 3 Effects of quercetin on serum creatinine and blood urea nitrogen after administration for 10 weeks. $* * P<0.01$, compared with non-diabetic control group; ${ }^{\#} P<0.05$, compared with either LD-quercetin or diabetic control groups.

Abbreviations: LD-quercetin, low dose quercetin; HD-quercetin, high dose quercetin; ctrl, control.

levels of UTP and UACR were still significantly higher than those observed in non-diabetic controls at the end of study, indicating that quercetin reduces glomerular hyperfiltration and ameliorates the functional abnormality of diabetic mice.

\section{Effect of quercetin on kidney weight and kidney index}

The kidney weights were significantly heavier and the kidney index was lower in $\mathrm{db} / \mathrm{db}$ mice as compared with $\mathrm{db} / \mathrm{m}$ mice $(P<0.05$, Figure 5A and B). In comparison with diabetic controls, the quercetin not only decreased the kidney weights but also improved the kidney index $(P<0.05$, Figure $5 \mathrm{~A}$ and $\mathrm{B})$, suggesting that quercetin has a remarkable effect on kidney index levels.

\section{Effect of quercetin on the serum lipid profile}

Serum lipids in $d b / d b$ and $d b / m$ mice during the same time-span were measured at the end of the study. It was indicated that average levels of serum cholesterol, triglycerides, and LDL-C were significantly higher in the $\mathrm{db} / \mathrm{db}$ mice in comparison with the $\mathrm{db} / \mathrm{m}$ mice $(P<0.01$, Table 1$)$, and the enhancement was dramatically blunted in those receiving low dose quercetin $(P<0.05$, Table 1$)$. Notably, the corresponding values further declined with an increased dose of quercetin. In contrast, a lower level of HDL-C was detected in the $\mathrm{db} / \mathrm{db}$ mice compared with the $\mathrm{db} / \mathrm{m}$ mice $(P<0.05$, Table 1$)$, but the level of HDL-C in high dose quercetin group was not remarkably different 

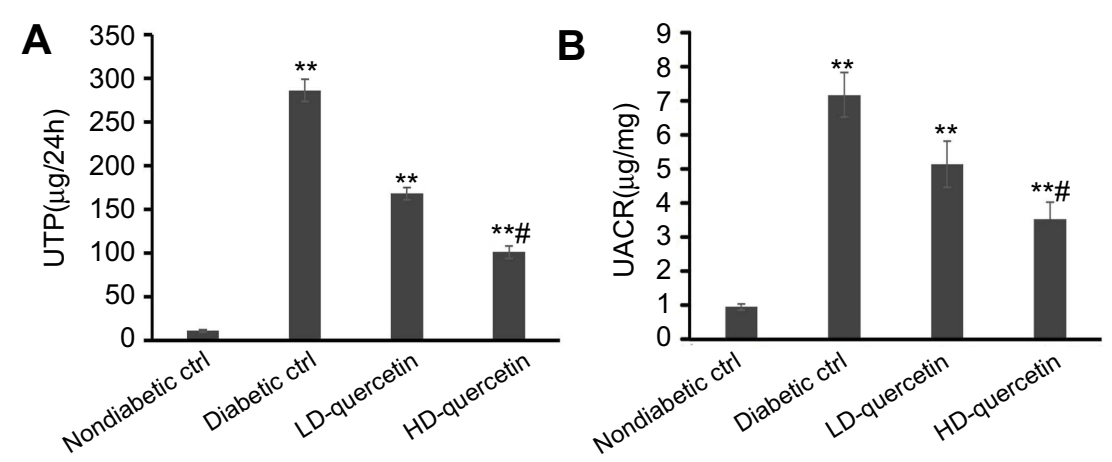

Figure 4 Effects of quercetin on hyperfiltration and microalbuminuria after administration for 10 weeks. (A) 24-hour UTP; (B) UACR. **P<0.0I, compared with nondiabetic control group; ${ }^{\#} P<0.05$, compared with either LD-quercetin group or diabetic control group.

Abbreviations: UTP, urine total protein; UACR, urinary albumin-to-creatinine ratio; LD-quercetin, low dose quercetin; HD-quercetin, high dose quercetin; ctrl, control.

A

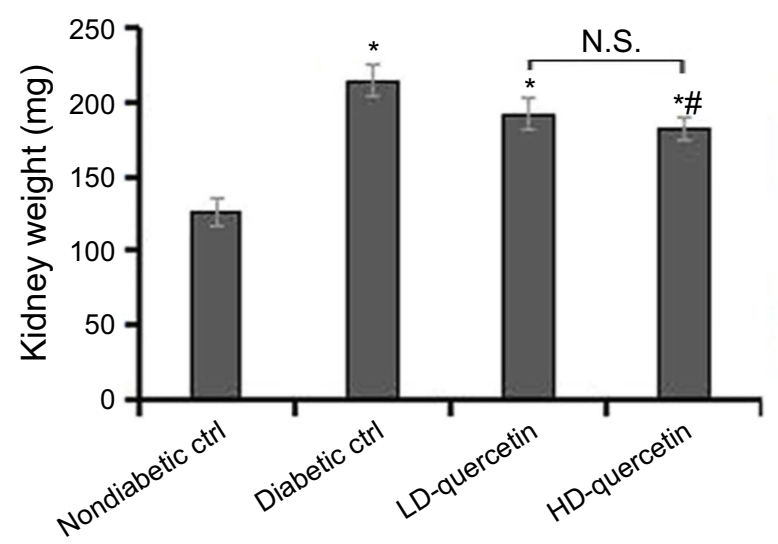

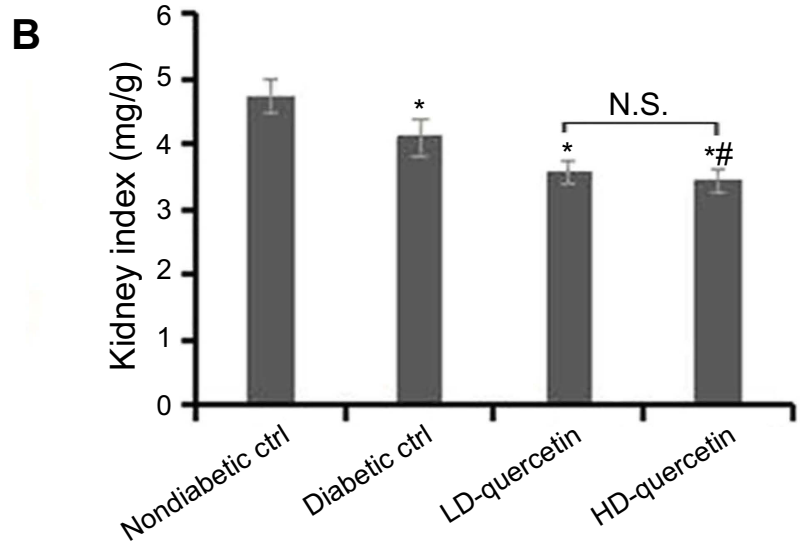

Figure 5 Effect of quercetin on the kidneys of mice after administration for 10 weeks. (A) The kidney weight of mice; (B) the kidney index of mice. $* P<0.05$, compared with non-diabetic control group; ${ }^{\#} P<0.05$, compared with diabetic control group.

Abbreviations: LD-quercetin, low dose quercetin; HD-quercetin, high dose quercetin; NS, no significant comparison; ctrl, control.

Table I Effect of quercetin on the blood lipid profile after administration for 10 weeks

\begin{tabular}{|l|l|l|l|l|}
\hline Parameters & Db/m & Db/db & Db/db+ LD-quercetin & Db/db+ HD-quercetin \\
\hline TC (mmol/L) & $1.51 \pm 0.20$ & $4.48 \pm 1.18^{* *}$ & $2.53 \pm 0.56^{\#}$ & $1.61 \pm 0.49^{\#, \ldots \#}$ \\
TG (mmol/L) & $0.67 \pm 0.22$ & $3.04 \pm 0.45^{* *}$ & $1.27 \pm 0.41^{\#}$ & $0.81 \pm 0.34^{\#, \ldots \#}$ \\
LDL-C (mmol/L) & $0.42 \pm 0.13$ & $2.84 \pm 0.25^{* *}$ & $0.87 \pm 0.34^{\#}$ & $0.46 \pm 0.28^{\#, \ldots \#}$ \\
HDL-C (mmol/L) & $0.98 \pm 0.27$ & $0.58 \pm 0.19^{*}$ & $0.63 \pm 0.25$ & $0.69 \pm 0.31$ \\
\hline
\end{tabular}

Notes: ${ }^{* * P}<0.01$ or $* P<0.05$, compared with non-diabetic control group; ${ }^{\#} P<0.01$, compared with diabetic control group; ${ }^{\#} P<0.05$, compared with LD-quercetin groups. Abbreviations: TC, total cholesterol; TG, triglycerides; HDL-C, high-density lipoprotein cholesterol; LDL-C, low-density lipoprotein cholesterol; LD-quercetin, low dose quercetin; HD-quercetin, high dose quercetin.

from that in both diabetic control and low dose quercetin groups $(P>0.05$, Table 1). Collectively, quercetin alleviates the impairment of lipid metabolism in diabetic mice.

\section{Effect of quercetin on histological alteration in renal tissue}

H\&E-stained sections from all groups showed histological changes with varying degrees of intensity. It depicted normal architectures (intact and clear glomerular basement membrane and tubules) and open glomerular capillary loops without any lesions in the normal non-diabetic $\mathrm{db} / \mathrm{m}$ mice (Figure 6A). Whereas renal tissue from diabetic control mice showed hypertrophic glomerular form, increased glomerular volume and glomerular basement membrane thickening, and mild mesangial matrix expansion (Figure 6B). All of the early glomerular pathological changes mentioned in the diabetic control group were obviously ameliorated in mice treated with high dose quercetin (Figure 6D), whereas the improvement was slightly smaller in low dose quercetin group (Figure 6C), as compared 
A

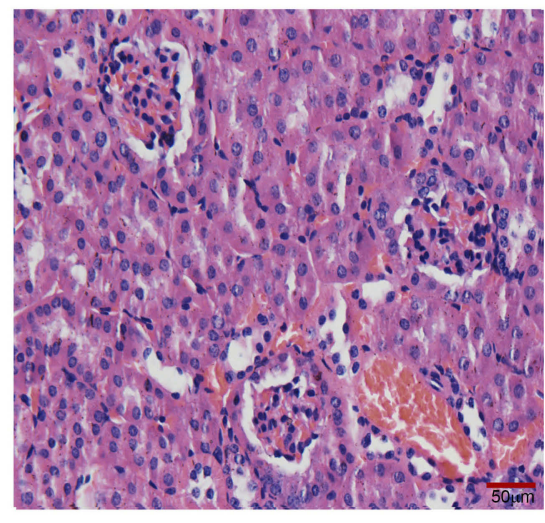

C

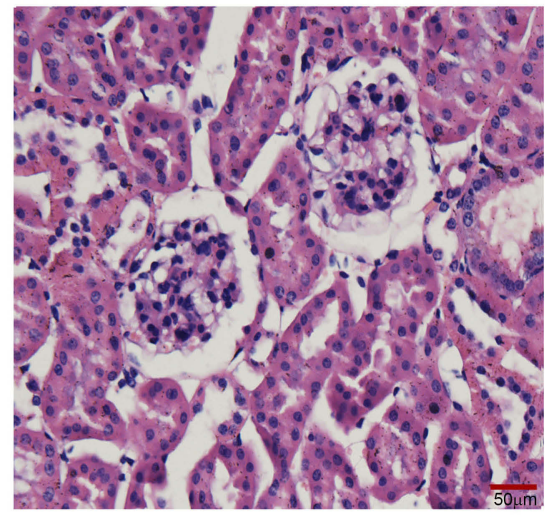

B

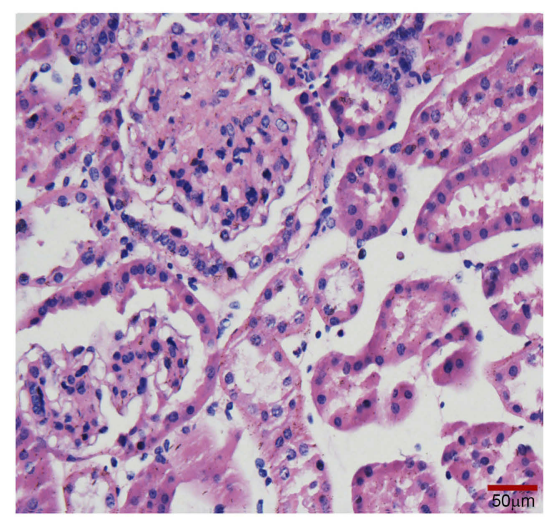

D

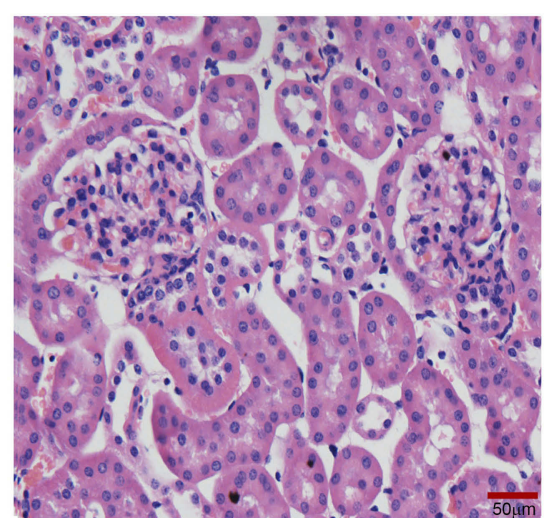

Figure 6 Effect of quercetin on renal structural alterations after administration for 10 weeks. Representative photomicrographs of $\mathrm{H} \& \mathrm{E}$ staining in kidney sections $($ bar $=50$ $\boldsymbol{\mu m}$ in the field). (A) $\mathrm{db} / \mathrm{m}$ mice; (B) $\mathrm{db} / \mathrm{db}$ control mice; (C) LD-quercetin treated $\mathrm{db} / \mathrm{db}$ mice; (D) HD-quercetin treated $\mathrm{db} / \mathrm{db}$ mice.

Abbreviations: LD-quercetin, low dose quercetin; HD-quercetin, high dose quercetin.

with diabetic controls. Collectively, quercetin improves the early glomerular pathological features and attenuates the progression of $\mathrm{DN}$ in $\mathrm{db} / \mathrm{db}$ mice.

\section{Effect of quercetin on lipid accumulation in the kidneys of mice}

Oil-Red-O staining was performed to help reveal cellular fat deposits. It was indicated that the presence of neutral lipids was observed in the renal tissue section of $\mathrm{db} / \mathrm{db}$ mice, not obviously in $\mathrm{db} / \mathrm{m}$ mice. There were excessive amounts of fat droplets of different sizes in the renal section from untreated $\mathrm{db} / \mathrm{db}$ mice, and the administration of quercetin reversed these changes in renal tissues of $\mathrm{db} /$ $\mathrm{db}$ mice with an increasing dose of quercetin $(P<0.05$, Figure 7), suggesting that quercetin attenuates renal lipid accumulation in the kidneys of diabetic mice.

\section{Effect of quercetin on the SCAP-SREBP2- LDLr signaling pathway}

The expression of LDLr, SREBP2, and SCAP in renal tissues was dramatically higher in diabetic control group than those in non-diabetic control group after administration for 10 weeks, and the differences were statistically significant (Figure 8A). Compared with diabetic controls, the expression level of LDLr, SREBP2, and SCAP proteins in the renal tissue of $\mathrm{db} / \mathrm{db}$ mice was significantly down-regulated after treatment with quercetin for 10 weeks, and the differences were statistically significant $(P<0.01$ or $P<0.05$, Figure $8 \mathrm{~B})$, confirming the downregulated effect of quercetin on the relative expression of key proteins in the SCAP-SREBP2-LDLr signaling pathway. Consistent with the down-regulation of renal LDLr, SREBP2, and SCAP expression, the renal expression of HMGCR protein was down-regulated with an increasing dose of quercetin in $\mathrm{db} / \mathrm{db}$ mice as compared with diabetic controls after treatment for 10 weeks, and the differences were statistically significant $(P<0.05$, Figure $8 \mathrm{~B})$.

\section{Discussion}

Although there are many novel drugs for DM, there are no specific curative treatments yet for DN. The use of natural substances for therapeutic purposes is currently 

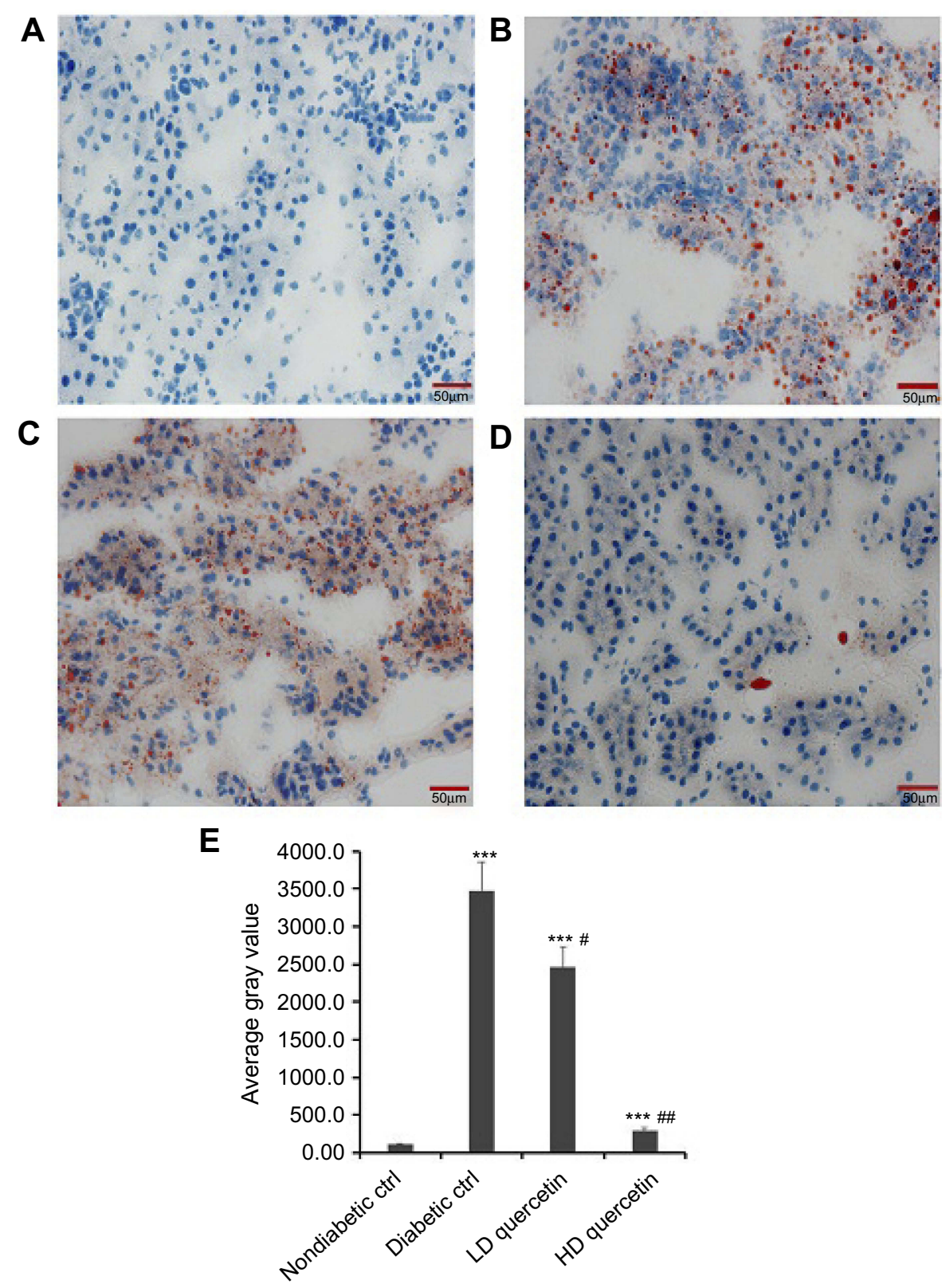

Figure 7 Effect of quercetin on the lipid accumulation in renal sections after administration for 10 weeks. Representative photomicrographs of $O i l-R e d-O$ staining (bar $=50$ $\mu \mathrm{m}$ in the field) in frozen kidney sections from $\mathrm{db} / \mathrm{m}$ mice (A), $\mathrm{db} / \mathrm{db}$ control mice (B), LD-quercetin treated db/db mice (C), and HD-quercetin treated db/db mice (D); (E) average gray value in different groups. The data obtained from three independent experimental studies are presented as the mean \pm SD. $* * * P<0.00 \mathrm{I}$, compared with both non-diabetic groups; ${ }^{\#} P<0.05$ or ${ }^{\# \#} P<0.01$, compared with diabetic control group.

Abbreviations: LD-quercetin, low dose quercetin; HD-quercetin, high dose quercetin; ctrl, control.

showing an increasing trend in human medicine, specifically, natural dietary compounds have received increasing attention for their ability to prevent or delay the development of pathogenesis during the early clinical manifestations of DM. Indeed, patients with diabetic kidney diseases in China have been widely receiving Chinese herbal medicine therapy. ${ }^{17}$ Notably, quercetin, as a chief member of flavonoids in TCM, has a potential ability to prevent the pathogenesis of DM in a dose-dependent manner to some extent. A previous study has already indicated that dietary quercetin significantly reduced body weight gain in high-fat diet-fed mice. ${ }^{30}$ In this study, quercetin did not exert a marked influence on body weight, food and liquid consumption, but it lowered the body weight gains in $\mathrm{db} / \mathrm{db}$ mice. Of note, the lack of obvious side effects of quercetin in our study is in line with the published observation that oral administration of quercetin to rats at doses up to $2,000 \mathrm{mg} / \mathrm{kg} / \mathrm{day}$ is 
A

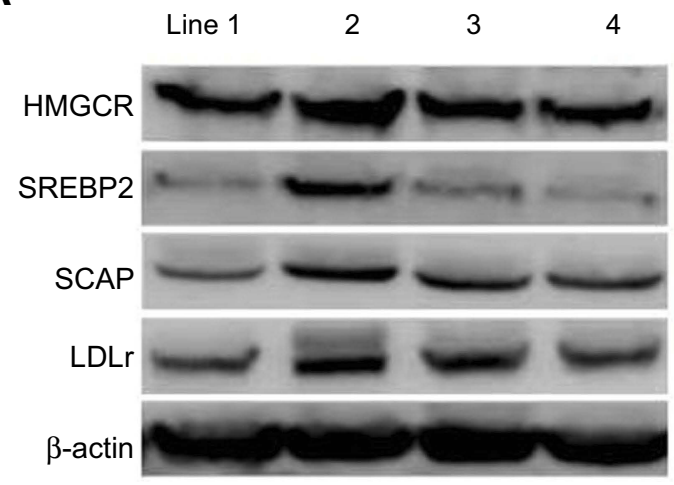

B

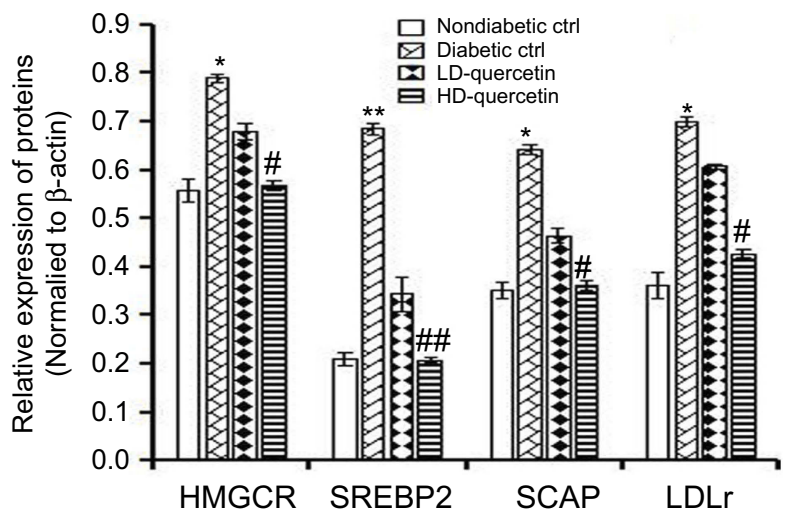

Figure 8 Effect of quercetin on the expression of key proteins in the SCAP-SREBP2-LDLr signaling pathway. (A) Diabetic db/db and non-diabetic db/m mice were administered either quercetin or saline for 10 weeks, and then the expression of key proteins was detected using Western blot analysis. Images shown are representative profiles from three technical replicates. (B) Relative expression of key proteins in renal tissues. The data obtained from three independent experimental studies are presented as the mean \pm SEM. If the error bar is not present, it is smaller than the thickness of graph line. ${ }^{*} P<0.05$ or $* * P<0.01$, compared with non-diabetic control group; ${ }^{*} P<0.05$ or ${ }^{\# \#} P<0.01$, compared with diabetic control group. Line I, 2, 3, and 4 represent non-diabetic control, diabetic control, LD-quercetin, and HD-quercetin groups, respectively. Abbreviations: LD-quercetin, low dose quercetin; HD-quercetin, high dose quercetin; ctrl, control.

considered to be safe. ${ }^{31}$ As is known, high level of blood sugar in diabetic patients causes severe lesions in the renal blood vessels due to too much stress on kidneys, causing development of acute and chronic kidney diseases and ultimately leading to kidney failure. ${ }^{32}$ In our present study, the mouse model not only had stable blood glucose in the range of $17.3 \sim 21.5 \mathrm{mmol} / \mathrm{L}$, but also had typical pathological features of early stage DN at 16 weeks of age. Thus, it is necessary to control blood sugar in diabetic patients or mouse models to prevent or attenuate DM-related early stage nephropathy. Using diabetic $\mathrm{db} / \mathrm{db}$ mice in our present study, the continuously increased blood glucose concentration was attenuated by quercetin as compared with control groups. In agreement with our results, a previous report by Jeong et al suggested that quercetin offered at $0.04 \%$ of the diet was enough to lower the level of blood glucose in $\mathrm{db} / \mathrm{db}$ mice relative to the control group. ${ }^{24}$ Considering these findings, quercetin by itself appears to be of great potential interest for preventing and treating DM-induced nephropathy.

Clinical characteristics of $\mathrm{DN}$ are progressive albuminuria and gradual decline of renal function. ${ }^{33}$ As demonstrated in a recent study, diabetic rats showed a significant decline in renal function and severe renal fibrosis under persistent diabetic conditions for 14 weeks, and administration with quercetin, although showing no remarkable effects on kidney index levels, dramatically alleviated the decline in the renal function of diabetic rats. ${ }^{34}$ In contrast to the previous findings, ours presented that kidney weights were significantly heavier and the kidney index was lower in $\mathrm{db} / \mathrm{db}$ mice, and administration of quercetin not only decreased kidney weights but also improved kidney index in comparison with diabetic controls. Notably, quercetin remarkably improved the signals of renal damage and dysfunction that were reflected by a reduction in serum creatinine, blood urea nitrogen, 24-hour urine protein, and UACR in diabetic mice. These concepts are supported by a previous study that similarly showed a remarkable effect of quercetin on improving the renal function in diabetic nephropathic rats. ${ }^{35}$ Collectively, these observations demonstrate that the lowering effect of quercetin on glomerular hyperfiltration may improve renal function and diabetic kidney injury.

Dyslipidemia is one of the most serious and frequently occurring complications in patients with DM and is greatly associated with DN. Being prone to developing dyslipidemia, the mice with uncontrolled lipid profile abnormalities are at the highest risk to develop DN. ${ }^{36}$ It has been demonstrated that dyslipidemia, a major risk factor in type $2 \mathrm{DM}$, is one of the most serious and frequently occurring complications characterized by abnormal levels of HDL-C, LDL-C, cholesterol, and triglycerides. ${ }^{37}$ Despite the lack of effect on body weight, the average level of serum cholesterol, triglycerides, and LDL-C in our current study was significantly higher in the diabetic mice in comparison with $\mathrm{db} / \mathrm{m}$ mice. More importantly, the enhancement was dramatically blunted in $\mathrm{db} / \mathrm{db}$ mice after administration of quercetin. In contrast, a lower level of HDL-C was detected in $\mathrm{db} / \mathrm{db}$ mice compared with $\mathrm{db} / \mathrm{m}$ mice, and the level of HDL-C in low dose quercetin group was not remarkably different from that in either diabetic control or high dose quercetin groups. In 
agreement with our results, Nekohashi et al indicated that quercetin significantly decreased the total-, free-, and LDL-C levels in the serum of Wistar rats. ${ }^{38}$ Conversely, it has been reported that either low or high doses of quercetin (52 or $105 \mathrm{mg} / \mathrm{kg} /$ day, respectively) improved hypertriglyceridemia, whereas only high dose of quercetin alleviated hypercholesterolemia and elevated HDL-C. ${ }^{24}$ The reasons for these differences might reflect species-specific responses to the quercetin. As a matter of fact, almost all patients with DM seem to develop lipid profile abnormalities, ultimately leading to build up dyslipidemia characterized by high serum levels of cholesterol, triglycerides and LDL-C and a reduced level of HDL-C. ${ }^{39}$ Therefore, it seems reasonable to assume that quercetin may be a lipid-lowering strategy to retard the progression of renal in diabetic mice.

Early stage DN is characterized histologically by glomerular hypertrophy, gradual thickening of glomerular basement membrane, and mild mesangial expansion, ${ }^{40,41}$ in which the enlarged glomeruli can increase the total surface area for filtration. Our results suggested that administration of quercetin to $\mathrm{db} / \mathrm{db}$ mice attenuated enlarged glomeruli and thickening of glomerular basement membrane, contributing to the decreased albuminuria in $\mathrm{db} / \mathrm{db}$ mice. Moreover, increasing cholesterol uptake and synthesis can result in lipid deposition in the kidneys. This can, in turn, cause cytotoxicity to glomerular and tubular cells, finally contributing to the progression of renal disease. In previous studies, ectopic lipid accumulation in the kidney has also been proposed to play a role in the pathogenesis of DN. ${ }^{8,9}$ Of note, there is a growing body of evidence showing the fact that quercetin alleviated kidney lipid accumulation in streptozotocin-induced DN rats, ${ }^{23}$ fructose-fed rats, ${ }^{42}$ and obese Zucker rats. ${ }^{43}$ These results are mostly consistent with the findings in animal models of diabetic kidney disease, in which accumulation of lipids, lipotoxicity, and lipid metabolism dysregulation are associated with renal damage. $^{44}$ Our present study found that there were excessive amounts of lipid droplets of different sizes in the kidney cortex of diabetic $\mathrm{db} / \mathrm{db}$ mice, as demonstrated by Oil-Red-O staining, and the lipid accumulation in both glomeruli and tubular cells of the kidneys could be effectively reversed by quercetin. Consistent with the Oil-Red-O staining, our results also demonstrated that the higher serum cholesterol and triglyceride content were significantly decreased in $\mathrm{db} / \mathrm{db}$ mice by quercetin. All these alternations correspond to the dramatically elevated cholesterol and triglyceride content of the kidney. Additionally, it is an indisputable and yet remarkable fact that despite persistent hyperglycemia, mesangial matrix expansion was prevented and the glomerular filtration rate was preserved in diabetic mice, as assessed by blood urea nitrogen, serum creatinine, and the UACR. All these findings imply that overt nephropathy was prevented in our study. Notably, quercetin protected the animal models of diabetic kidney disease without causing any noticeable systemic side effects. Therefore, our data and other strongly provided convincing evidence that quercetin may play an unexpected role in regulating lipid metabolism and attenuating the early pathological glomerular changes.

Despite the increased knowledge of the benefits of quercetin, the mechanisms underlying the protective effect of quercetin on diabetic kidney disease might be complicated. Recently, a growing body of evidence has demonstrated that quercetin effectively affected various glucose and lipid metabolism disorders through multi-pathway mechanisms including Akt signaling and SIRT1, ${ }^{23}$ PI3K/AKT pathway, ${ }^{45}$ and endoplasmic reticulum stress pathway. ${ }^{46}$ It has also been demonstrated that increasing HMGCR-mediated cholesterol synthesis and LDLr-mediated cholesterol uptake possibly resulted in renal cholesterol accumulation in diabetic rats. ${ }^{16}$ Except for oxidative process of quercetin, another hypothesis has also been provided: that the deleterious effect of LDL-C and VLDL-C could be prevented by decreasing HMGCA. ${ }^{47}$ The decrease of renal HMGCR expression paralleled the significant lowering of serum triglycerides, cholesterol, and LDL-C levels in $\mathrm{db} / \mathrm{db}$ mice after treatment with quercetin for 10 weeks, as compared with diabetic controls in our current study. These data are consistent with the reduction in renal lipid accumulation observed in Oil-red-O staining. Clinical studies have indicated that the inhibition of cholesterol biosynthesis to reduce de novo synthesis of cholesterol by HMGCA reductase inhibitors (statins), protected against diabetic and non-diabetic renal disease. ${ }^{48,49}$ Thus, decreasing HMGCR-mediated cholesterol synthesis is conductive to the renal cholesterol accumulation in diabetic mice. In addition, animal and clinical studies have reported the roles of LDLrSREBP2-SCAP signaling pathway in early stage DN, and the blockade of these pathways slows the progression and development of DN. For example, using type 2 diabetic $\mathrm{db} /$ $\mathrm{db}$ mice and cell culture model of podocytes, Zhang et al found that, through the disruption of LDLr pathway, lipid accumulation in kidneys and in podocytes was increased under high glucose stimulation. ${ }^{6}$ Similarly, Sun et al found significant lipid accumulation and up-regulation of LDLr, SCAP, and SREBP-2 expressions in the kidneys of diabetic rats. ${ }^{16}$ Our present results confirmed that the renal expression 
of key proteins, such as LDLr, SREBP2, and SCAP, was dramatically higher in diabetic control group compared with non-diabetic control group, and the higher level of corresponding values was significantly down-regulated in diabetic mice after administration for 10 weeks as compared with diabetic controls. These concepts are consistent with the findings of other studies that dysregulation of cholesterol metabolism has been linked to lipotoxicity and lipid accumulation in early stage DM through the disruption of LDLr pathway. ${ }^{6,16}$ Additionally, a previous study has demonstrated that SCAP/SREBP pathway is also essential for developing diabetic fatty liver disease in animals. ${ }^{50}$ Thus, whether quercetin improves lipid metabolism disorder in the liver via SCAP/SREBP pathway should be further studied in the future.

In summary, the findings of the current study confirm the protective effect of quercetin against the initiation and progression of $\mathrm{DN}$ in diabetic mice by improving the renal accumulation of lipid bodies. It also seems that a renoprotective effect of quercetin is, at least in part, mediated through SCAP-SREBP2-LDLr signaling pathway, resulting in the alleviation of DM-associated renal injury.

\section{Acknowledgments}

This study was supported by the Fund for Traditional Chinese Medicine Science and Technology Project of Jiangsu Province (YB2015104). The supporting organization did not participate in the study design, data collection, analysis, and interpretation. The authors would like to thank Dr Feng Gao (Medical College of Southeast University) for technical assistance, and the authors also thank Jian Gu (Department of Laboratory Medicine, Affiliated Hospital of Nanjing University of Chinese Medicine, Nanjing, China) for his technical assistance and instructions.

\section{Author contributions}

All authors contributed to data analysis, drafting or revising the article, gave final approval of the version to be published, and agree to be accountable for all aspects of the work.

\section{Disclosure}

The authors report no conflicts of interest in this work.

\section{References}

1. Guariguata L, Whiting DR, Hambleton I, Beagleya J, Linnenkampa U, Shaw JE. Global estimates of diabetes prevalence for 2013 and projections for 2035. Diabetes Res Clin Pract. 2014;103 (2):137-149. doi:10.1016/j.diabres.2013.11.002

2. Figueroa-Pérez MG, Pérez-Ramírez IF, Enciso-Moreno JA, GallegosCorona MA, Salgado LM, Reynoso-Camacho R. Diabetic nephropathy is ameliorated with peppermint (Mentha piperita) infusions prepared from salicylic acid-elicited plants. J Funct Foods. 2018;43:55-61. doi:10.1016/j.jff.2018.01.029

3. UK Prospective Diabetes Study (UKPDS) Group. Intensive blood-glucose control with sulphonylureas or insulin compared with conventional treatment and risk of complications in patients with type 2 diabetes (UKPDS 33). Lancet. 1998;352(9131):837-853.

4. Khangholi S, Majid FA, Berwary NJ, Ahmad F, Aziz RB. The mechanisms of inhibition of advanced glycation end products formation through polyphenols in hyperglycemic condition. Planta Med. 2016;82(1-2):32-45. doi:10.1055/s-0035-1558086

5. Tsai SF, Su CW, Wu MJ, et al. Urinary cyclophilin A as a new marker for diabetic nephropathy: a cross-sectional analysis of diabetes mellitus. Medicine. 2015;94(42):e1802. doi:10.1097/MD.0000000000000874

6. Zhang Y, Ma KL, Liu J, et al. Inflammatory stress exacerbates lipid accumulation and podocyte injuries in diabetic nephropathy. Acta Diabetol. 2015;52(6):1045-1056. doi:10.1007/s00592-015-0753-9

7. Mount P, Davies M, Choy S-W, Cook N, Power D. Obesity-related chronic kidney disease - the role of lipid metabolism. Metabolites. 2015;5(4):720-732. doi:10.3390/metabo5040720

8. Yuan Y, Sun H, Sun ZL. Advanced glycation end products (AGEs) increase renal lipid accumulation: apathogenic factor of diabetic nephropathy (DN). Lipids Health Dis. 2017;16(1):126. doi:10.1186/s12944-0170565-8

9. Herman-Edelstein M, Scherzer P, Tobar A, Levi M, Gafter U. Altered renal lipid metabolism and renal lipid accumulation in human diabetic nephropathy. J Lipid Res. 2014;55(3):561-572. doi:10.1194/jlr.P040501

10. Allen TJ, Cooper ME, Lan HY. Use of genetic mouse models in the study of diabetic nephropathy. Curr Diab Rep. 2004;4(6):435-440.

11. de Boer IH. A new chapter for diabetic kidney disease. $N$ Engl J Med. 2017;377(9):885-887. doi:10.1056/NEJMe1708949

12. D'Addio F, Trevisani A, Ben Nasr M, et al. Harnessing the immunological properties of stem cells as a therapeutic option for diabetic nephropathy. Acta Diabetol. 2014;51(6):897-904. doi:10.1007/s00592-014-0603-1

13. Rice LM, Donigan M, Yang M, et al. Protein phosphatase 2A (PP2A) regulates low density lipoprotein uptake through regulating sterol response element-binding protein-2 (SREBP-2) DNA binding. $J$ Biol Chem. 2014;289(24):17268-17279. doi:10.1074/jbc.M114.570390

14. Vallett SM, Sanchez HB, Rosenfeld JM, Osborne TF. A direct role for sterol regulatory element binding protein in activation of 3-hydroxy-3-methylglutaryl coenzyme A reductase gene. $J$ Biol Chem. 1996;271(21):12247-12253.

15. Brown MS, Goldstein JL. A receptor-mediated pathway for cholesterol homeostasis (Nobel lecture). Angew Chem Int Ed. 1986;17(45):34-47.

16. Sun H, Yuan Y, Sun Z-L. Cholesterol contributes to diabetic nephropathy through SCAP- SREBP-2 pathway. Int $J$ Endocrinol. 2013;2013:1-8. doi:10.1155/2013/592576

17. Sun GD, Li CY, Cui WP, et al. Review of herbal traditional Chinese medicine for the treatment of diabetic nephropathy. J Diabetes Res. 2016;2016:1-18.

18. Wen X, Zeng Y, Liu L, et al. Zhenqing recipe alleviates diabetic nephropathy in experimental type 2 diabetic rats through suppression of SREBP-1c. J Ethnopharmacol. 2012;142(1):144-150. doi:10.1016/j. jep.2012.04.028

19. D'Andrea G. Quercetin: a flavonol with multifaceted therapeutic applications? Fitoterapia. 2015;106:256-271. doi:10.1016/j. fitote.2015.09.018 
20. Khan F, Niaz K, Maqbool F, et al. Molecular targets underlying the anticancer effects of quercetin: an update. Nutrients. 2016;8(9):529. doi:10.3390/nu8090529

21. Miltonprabu S, Tomczyk M, Skalicka-Woźniak K, et al. Hepatoprotective effect of quercetin: from chemistry to medicine. Food Chem Toxicol. 2017;108(Part B):365-374. doi:10.1016/j.fct.2016.08.034

22. Li XL, Zhou N, Wang J, et al. Quercetin suppresses breast cancer stem cells $\left(\mathrm{CD} 44^{+} / \mathrm{CD} 24^{-}\right)$by inhibiting the PI3K/Akt/mTOR-signaling pathway. Life Sci. 2018;196:56-62. doi:10.1016/j.1fs.2018.01.014

23. Peng J, Li Q, Li K, et al. Quercetin improves glucose and lipid metabolism of diabetic rats: involvement of Akt signaling and SIRT1. J Diabetes Res. 2017;2017:1-10. doi:10.1155/2017/3417306

24. Jeong S-M, Kang M-J, Choi H-N, Kim J-H, Kim J-I. Quercetin ameliorates hyperglycemia and dyslipidemia and improves antioxidant status in type 2 diabetic db/db mice. Nutr Res Pract. 2012;6 (3):201-207. doi:10.4162/nrp.2012.6.3.201

25. Guo XP, Chen M, Zeng HM, et al. Quercetin attenuates ethanol-inducediron uptake and myocardial injury by regulating the angiotensin II-L-type calcium channel. Mol Nutr Food Res. 2018;62:1700772. doi:10.1002/mnfr.201700879

26. Yuan YY, Ge SN, Lv ZH, et al. Attenuation of perfluorooctanoic acid-induced testicular oxidative stress and apoptosis by quercetin in mice. RSC Adv. 2017;7:45045-45052. doi:10.1039/C7RA09036F

27. Zhou L, An X-F, Teng S-C, et al. Pretreatment with the total flavone glycosides of flos abelmoschus manihot and hyperoside prevents glomerular podocyte apoptosis in streptozotocin-induced diabetic nephropathy. J Med Food. 2012;15(5):461-468. doi:10.1089/jmf.2011.1921

28. Ge J, Miao J-J, Sun X-Y, Yu J-Y. Huangkui capsule, an extract from Abelmoschus manihot (L.) medic, improves diabetic nephropathy via activating peroxisome proliferator-activated receptor (PPAR)- $\alpha / \gamma$ and attenuating endoplasmic reticulum stress in rats. J Ethnopharmacol. 2016;189:238-249. doi:10.1016/j.jep.2016.05.033

29. Bindesbøll C, Berg O, Arntsen B, Nebb HI, Dalen KT. Fatty acids regulate perilipin5 in muscle by activating PPAR $\delta$. J Lipid Res. 2013;54(7):1949-1963. doi:10.1194/jlr.M038992

30. Dong J, Zhang X, Zhang L, et al. Quercetin reduces obesity-associated ATM infiltration and inflammation in mice: amechanism including AMPKa1/SIRT1. J Lipid Res. 2014;55:363-374. doi:10.1194/jlr. M038786

31. Harwood M, Danielewskanikiel B, Borzelleca JF, Flamm GW, Williams GM, Lines TC. A critical review of the data related to the safety of quercetin and lack of evidence of in vivo toxicity, including lack of genotoxic/carcinogenic properties. Food Chem Toxicol. 2007;45(11):2179-2205. doi:10.1016/j.fet.2007.05.015

32. Lv M, Chen Z, Hu G, Li Q. Therapeutic strategies of diabetic nephropathy: recentprogress and future perspectives. Drug Discov Today. 2015;20(3):332-346. doi:10.1016/j.drudis.2014.10.007

33. American Diabetes Association. Standards of medical care in diabetes-2010. Diabetes Care. 2010;33(Suppl 1):S11-S61. doi:10.2337/ dc10-S011

34. Lu Q, Ji X-J, Zhou Y-X, et al. Quercetin inhibits the mTORC1/ p70S6K signaling-mediated renal tubular epithelial-mesenchymal transition and renal fibrosis in diabetic nephropathy. Pharmacol Res. 2015;99:237-247. doi:10.1016/j.phrs.2015.06.006

35. Lai P-B, Zhang L, Yang L-Y. Quercetin ameliorates diabetic nephropathy by reducing the expressions of transforming growth factor- $\beta 1$ and connective tissue growth factor in streptozotocin-induced diabetic rats. Ren Fail. 2012;34(1):83-87. doi:10.3109/0886022X.2011.623564
36. Debendra NR, Islam MD, Biswas M, Sana S, Debendra S, Roy N. Dyslipidemia in diabetes patient is responsible for developing nephritic syndrome with increasing ages, a survey based cross sectional study. Int J Biomed Res. 2016;7:652-657.

37. Tangvarasittichai S. Oxidative stress, insulin resistance, dyslipidemia and type 2 diabetes mellitus. World J Diabetes. 2015;6(3):456-480. doi:10.4239/wjd.v6.i3.456

38. Nekohashi M, Ogawa M, Ogihara T, et al. Luteolin and quercetin affect the cholesterol absorption mediated by epithelial cholesterol transporter niemann-pick C1-like 1 in Caco-2 cells and rats. PLoS One. 2014;9(5):e97901. doi:10.1371/journal.pone.0097901

39. Quiroga B, Arroyo D, de Arriba G. Present and future in the treatment of diabetic kidney disease. J Diabetes Res. 2015;2015:801348. doi: $10.1155 / 2015 / 815839$

40. Shaikh AS, Somani RS. Animal models and biomarkers of neuropathy in diabetic rodents. Indian J Pharmacol. 2010;42(3):129-134. doi:10.4103/0253-7613.66833

41. Zhang J, Wang Y, Gurung P, et al. The relationship between the thickness of glomerular basement membrane and renal outcomes in patients with diabetic nephropathy. Acta Diabetol. 2018;55 (7):669-679. doi:10.1007/s00592-018-1128-9

42. Hu Q-H, Zhang X, Pan Y, Li Y-C, Kong L-D. Allopurinol, quercetin and rutin ameliorate renal NLRP3 inflammasome activation and lipid accumulation in fructose-fed rats. Biochem Pharmacol. 2012;84 (1):113-125. doi:10.1016/j.bcp.2012.03.005

43. Rivera L, Morón R, Sánchez M, Zarzuelo A, Galisteo M. Quercetin ameliorates metabolic syndrome and improves the inflammatory status in obese Zucker rats. Obesity. 2008;16(9):2081-2087. doi:10.1038/ oby. 2008.315

44. Wang XX, Jiang T, Shen Y, et al. Diabetic nephropathy is accelerated by farnesoid $\mathrm{X}$ receptor deficiency and inhibited by farnesoid $\mathrm{X}$ receptor activation in a type 1 diabetes model. Diabetes. 2010;59 (11):2916-2927. doi:10.2337/db10-0019

45. Pisonero-Vaquero S, Martínez Ferreras Á, García-Mediavilla M, et al. Quercetin ameliorates dysregulation of lipid metabolism genes via the $\mathrm{PI} 3 \mathrm{~K} / \mathrm{AKT}$ pathway in a diet-induced mouse model of nonalcoholic fatty liver disease. Mol Nutr Food Res. 2015;59(5):879-893. doi:10.1002/ mnfr.201400913

46. Cai X, Bao L, Dai X, Ding Y, Zhang Z, Li Y. Quercetin protects RAW264.7 macrophages from glucosamine-induced apoptosis and lipid accumulation via the endoplasmic reticulum stress pathway. Mol Med Rep. 2015;12(5):7545-7553. doi:10.3892/ mmr.2015.4340

47. Seiva FR, Chuffa LG, Braga CP, Amorima JP, Fernandes AA. Quercetin ameliorates glucose and lipid metabolism and improves antioxidant status in postnatally monosodium glutamate-induced metabolic alterations. Food Chem Toxicol. 2012;50(10):3556-3561. doi:10.1016/j.fct.2012.07.009

48. Wang Z, Jiang T, Li J, et al. Regulation of renal lipid metabolism, lipid accumulation, and glomerulosclerosis in FVBdb/db mice with type 2 diabetes. Diabetes. 2005;54(8):2328-2335.

49. Istvan ES, Deisenhofer J. Structural mechanism for statin inhibition of HMG-CoA reductase. Science. 2001;292(5519):1160-1164. doi:10.1126/science. 1059344

50. Moon Y-A, Liang GS, Xie XF, et al. The Scap/SREBP pathway is essential for developing diabetic fatty liver and carbohydrate-induced hypertriglyceridemia in animals. Cell Metab. 2012;15(2):240-246. doi:10.1016/j.cmet.2011.12.017 


\section{Publish your work in this journal}

Diabetes, Metabolic Syndrome and Obesity: Targets and Therapy is an international, peer-reviewed open-access journal committed to the rapid publication of the latest laboratory and clinical findings in the fields of diabetes, metabolic syndrome and obesity research. Original research, review, case reports, hypothesis formation, expert opinion and commentaries are all considered for publication. The manuscript management system is completely online and includes a very quick and fair peer-review system, which is all easy to use. Visit http://www.dovepress.com/testimonials.php to read real quotes from published authors.

Submit your manuscript here: https://www.dovepress.com/diabetes-metabolic-syndrome-and-obesity-targets-and-therapy-journal 